Chapter 2

\title{
Cleft Lip and Palate Patients: Diagnosis and Treatment
}

\author{
Letizia Perillo, Fabrizia d'Apuzzo, Sara Eslami and \\ Abdolreza Jamilian
}

Additional information is available at the end of the chapter

http://dx.doi.org/10.5772/67328

\begin{abstract}
Cleft lip or palate is one of the most common types of craniomaxillofacial birth anomalies. Midface deficiency is a common feature of cleft lip and palate patients due to scar tissue of the lip and palate closure. Cleft lip and palate patients should be carefully evaluated by the craniofacial team in order to detect potentially serious deformities. Craniofacial team is involved with diagnosis of facial morphology, feeding problems, guidance of the growth and development of the face, occlusion, dentition, hearing and speech problems, and psychosocial issues and jaw discrepancy of the patients with cleft lip and palate or craniofacial syndromes. Treatment for cleft children requires a multidisciplinary approach including facial surgery in the first months of life, preventive and interceptive treatment in primary dentition, speech therapy, orthodontics in the mixed dentition phase, oromaxillofacial surgery, and implant and prosthetics in adults. Treatment plan from orthodontic perspective can be divided into the following stages based on the dentition stages: (1) presurgical orthopedics, (2) primary dentition, (3) mixed dentition, and (4) permanent dentition. The aim of this chapter is to assess a rational team work approach in the management of the patient with cleft lip and/or palate from birth to adulthood.
\end{abstract}

Keywords: cleft lip, cleft palate, diagnosis, treatment, maxillary deficiency

\section{Introduction}

Cleft lip or palate is one of the most common types of cranio-maxillofacial birth anomalies. It accounts for $65 \%$ of all head and neck deformities [1]. Maxillary deficiency is a common feature of cleft lip and palate patients due to scar tissue of the lip and palate closure. For treatment of maxillary deficiency, various devices, such as facemask [2], protraction headgear [3], orthopedic mask [4], reverse chin cup [5], tongue appliance [6], tongue plate [7], surgically assisted orthopedic protraction, and distraction osteogenesis have been introduced [8]. Treatment of 
cleft lip and palate in patients should be started after birth and continues to adulthood. Lip closure and palatal closure are performed at 3 months and around 12 months of age, respectively, as well as secondary alveolar bone graft is done between 9 and 12 years of age [9]. However, orthodontic intervention usually begins during the mixed dentition. Orthodontic treatment in patients with cleft lip and palate are focused on maxillary arch expansion, correction of upper incisor misalignments, gross rotations of incisors, and crossbites and correction of Class III skeletal growth pattern. Patients with cleft lip and/or palate should be treated by teamwork. The team conception allows a systematic treatment plan to be developed and allows the team members to work together properly to identify problems. Orthodontic treatment plan can be divided into the following stages based on the dentition stages: (1) presurgical orthopedics, (2) primary dentition, (3) mixed dentition, and (4) permanent dentition.

In the following sections about diagnosis, classification, and treatment options in different period of time are discussed. Therefore, the aim of this chapter is to assess a rational teamwork approach to the management of the patient with cleft lip and/or palate from birth to adulthood.

\section{Diagnostic considerations}

Ultrasonography serves as a noninvasive diagnostic tool, now regularly recommended as a routine component of prenatal consideration. This noninvasive diagnostic tool helps to determine gestational age, confirm fetal viability, check placental location, establish the growth and their number of fetuses, and examine fetal anatomy to detect any deformities [10].

\section{Treatment planning for cleft lip and palate patients}

The treatment of cleft lip and palate should be started right after birth. Treatment for cleft children requires a multidisciplinary approach including: facial surgery in the first months of life, preventive and interceptive treatment in primary dentition, speech therapy, orthodontics in the mixed dentition phase, oral and maxillofacial surgery, and implant and prosthetics in adults. So the treatment to achieve a proper occlusion and function often lasts from birth until adulthood. Patients with cleft lip and palate routinely require extensive and prolonged orthodontic treatment. Close cooperation between the orthodontist, surgeon, prosthodontist, and general dentist is required. Cleft lip and palate patients should be carefully evaluated by the craniofacial team in order to detect potentially serious deformities that can be associated with cleft lip and palate. The team conception allows a systematic treatment plan to be developed and allows the team members to work together properly to identify problems. Maxillary deficiency is a common developmental problem in cleft lip and palate patients. The treatment objectives are to correct the deficient maxillary arch, the anterior and posterior cross bites, correct misaligned maxillary incisors, and also obtain satisfactory overjet and overbite. The following general and local factors must be evaluated before planning any treatment. 


\subsection{General factors}

(a) Health of the patient includes physical, mental, and social.

(b) Family background and attitude.

\subsection{Local factors}

(a) Width of the cleft.

(b) Adequacy of tissue adjacent to the cleft.

(c) Length of soft palate in relation to nasopharynx.

(d) Configuration of nasopharynx.

(e) Functional activity of palate-pharyngeal muscles.

\subsection{Interceptive treatment}

Interceptive treatment is recommended because it can

- improve facial and dental esthetics,

- help overcome psychological issues,

- improve speech,

- reduce the risk of decay, and

- avoid the need for major surgery in the future.

\subsection{Cleft lip repair}

The aim of lip repair is to close the cleft to create esthetics of the face and to restore muscular anatomy of the upper lip. This procedure will serve to develop lip normally with minimal scar tissue. Closure of the lip is accomplished by the plastic or maxillofacial surgeon when the patient is approximately 3 months of age and weighs at least 10 pounds.

\subsection{Cleft palate repair}

The objective of cleft palate surgery is to close the palate to restore normal function to eating and drinking and to enhance the development of normal speech. The palate forms the floor of the nose and the roof of the oral cavity; thus, a cleft causes a free communication between these two cavities. Treatment of cleft lip and palatal patients is complex because of potential problems with middle ear infections, speech, feeding, occlusion, creating maxillary deficiency due to scar tissue from surgical procedure, and jaw abnormalities. Surgical procedure of the cleft palate is best performed before the child reaches 12 months of age. This procedure is called palatoplasty. Additional surgeries are often needed to achieve the best results [9]. 


\subsection{Nursing care}

The role of nursing in the care of patients and or families includes family education, case management, access to team care, assistance with infant feeding, consultation, research, and primary care.

\subsection{Feeding problems}

An infant with a cleft may have difficulty in feeding due to lack of suction. Cleft lip patients do not usually have feeding problems. But when the palate is involved, feeding can be a crucial challenge. Normally, the palate serves as a barrier to prevent food and liquids from entering the nose. A patient with cleft palate swallows a lot of air and regurgitates food into the nose. Cleft palate patients may need a special bottle and nipple to receive milk. Patients with feeding problems should be visited regularly by a specialist to make sure that they are gaining weight well.

\subsection{Speech problems}

Difficulty in speech is the number one issue for cleft patients after facial and dental esthetics. They have difficulty in speaking correctly, and treatment can help them to achieve a good tongue posture. Difficulty with speech articulation is common in cleft lip and palate patients. Many patients require speech therapy after surgery. Speech therapist will repeatedly evaluate speech development and will arrange for necessitate speech therapy.

\subsection{Dental problems}

Dental problems have an effect on chewing, facial esthetics, speech thus these patients frequently require orthodontic treatment. Prevalence of dental anomalies such as variations in tooth number, dislocation, missing, supernumerary, tooth shape, and reduced tooth dimensions has always been found to be higher in cleft lip and or palate patients when compared with general population [11]. Akcam et al. [12] reported that a significant amount $(96.7 \%)$ of children with cleft had at least one dental irregularity. Shapira et al. [13] found that in the cleft area, most developmental dental anomalies are related to the maxillary lateral incisor, both in the primary and in the permanent dentitions. Shape anomalies, such as enamel hypoplasia and peg shaped, have also been frequently seen in cleft lip and/or palate patients [14]. Tooth agenesis, known as hypodontia or congenital absence of teeth, is the most commonly detected developmental abnormality of the human dentition. All types of clefts are often associated with congenitally missing teeth [15]. Jiroutova and Mullerova [16] studied the frequency of hypodontia in cleft lip and or palate patients and found that the maxillary dentition was affected more often in cleft lip and palate, the mandible was involved more frequently in isolated cleft palate. The dental bud of the upper lateral incisor was affected most commonly in cleft lip and in cleft lip and palate, whereas the second lower premolar was most frequently absent in an isolated cleft palate. 
Paranaiba et al. studied prevalence of dental abnormalities in patients with nonsyndromic cleft lip and/or palate in Brazilian population and found that agenesis of the premolars and maxillary lateral incisors were significantly more frequent in patients with unilateral complete cleft lip and palate [17]. In various studies, lateral incisors were reported to be the most commonly missing teeth followed by second premolars [12, 18, 19]. While, in a another study Laatikainen and Ranta [20] found that the maxillary second premolar was the most frequently absent tooth, followed in order of frequency by the upper lateral incisor and the mandibular second premolar.

Polder et al. [21] did a meta-analysis of the prevalence of dental agenesis of permanent teeth of Caucasian populations in North America, Australia, and Europe and found that mandibular second premolar was the most affected tooth, followed by the upper lateral incisor and the upper second premolar. Shapira et al. [22] found a total of 47 missing second premolars in the upper arch and 23 missing in the mandible. In literature, Ranta [23] reported that the incidence of hypodontia rises strongly with the severity of cleft. Paranaiba et al. [17] also found that patients with unilateral cleft lip and palate were affected more significantly by dental anomalies than those with bilateral cleft lip and palate. It should be considered that ethnicity also plays an important role in prevalence of cleft and associated anomalies. Polder et al. reported that prevalence of dental agenesis in Europe and Australia was higher than in North America. In addition, they reported that the prevalence of dental agenesis in females is 1.37 times higher than in males for all three continents. Defects of enamel such as hypoplasia and opacities are common in the teeth adjacent to the cleft site. Decay is higher in these patients compared with the non-cleft populations. Therefore there is a higher incidence of caries in teeth with enamel defects, the issues of prevention and brushing are of great importance in cleft children [24].

\subsection{Role of psychologist in cleft lip and palate patients}

It is clear that attractive children are seen by others as happier, having more positive social behavior. Children with cleft may have a less attractive appearance and speech problems make it worst. Babies may face bullying and teasing because of their appearance. Parents may be more tolerant of misbehavior in their child and are more likely to spoil their child by being overprotective. Moreover, peer interaction also has an important role in maintaining psychosocial problems. Cleft lip and palate patients are at high risk for developing psychosocial problems especially those relating to self-concept, peer relationships, and appearance. Psychological problems affect development of children with cleft lip and palate. Therefore, these patients are treated by the interdisciplinary team to maximize positive outcome of treatment. Missing teeth, feeding difficulties, the infant's appearance, presence of misaligned teeth, and severe malocclusion can lead to social isolation. Moreover, frequent visits to the doctor and surgeries can be quite stressful. The psychologist provides treatment plan for developmental, emotional, learning, and adjustment abnormalities. They focused on the appearance, speech, patient's self-esteem, psychosocial problems, self-confidence, interpersonal relationships, and emotional handicapped problems [25]. 


\subsection{Role of otolaryngologist in cleft lip and palate patients}

Patients with cleft lip and palate have a higher incidence of hearing problems. They have more frequent problems with fluid, ear infections, and otitis media, which can be very painful. It is crucial that to have the infant's hearing tested during the first few months and it is also very important that cleft lip and palate patients have regular hearing tests to screen middle ear problems. This could adjust the development of normal hearing as well as speech. As the child gets older, the rate of ear infections seems to reduce. Any abnormality of the upper airway can affect the function of the Eustachian tube and enhance the possibility of persistent fluid in the middle ear, which is a major cause of repeated ear infections. Hearing loss can be a consequence of repeat ear infections and persistent middle ear fluid. Tubes can be inserted in the ear by an otolaryngologist to relieve fluid build-up and repair hearing. Cleft lip and palate patients are suffering from mouth breathing, feeding, hearing, speech problems, and jaw deformities. There are some areas of overlap with plastic surgery, oral and maxillofacial surgery, otolaryngology, and speech-language pathology. Oral and maxillofacial surgeon evaluates the skeletal discrepancies related to cleft and craniofacial abnormalities such as maxillary deficiency and other skeletal malocclusions. Surgeons work with other members of the group to ensure appropriate and harmonious facial form and dental arch.

\subsection{Role of pedodontist in cleft lip and palate patients}

The role of pediatric dentistry in treatment of cleft lip and palate cleft patients is the comprehensive preventative and therapeutic oral health care of children, counseling and caries control. These treatments include following steps:

(a) Growth and development monitoring.

(b) Caries prevention and oral hygiene guidance.

(c) Behavior modifications.

(d) Routine dental care.

(e) Preventive and interceptive dentistry.

(f) Interceptive orthodontics where appropriate.

(g) Restorative procedures.

(h) Removal of primary dentition in surgical site.

(i) Periodontal considerations.

\subsection{Role of orthodontist in cleft lip and palate patients}

In the management of cleft lip and palate patients the orthodontist has an important role in the cleft and craniofacial team. They are involved with diagnosis of facial morphology, guidance of the growth and development of the face, occlusion, dentition, and jaw discrepancy of the patients with cleft or craniofacial syndromes. They provide orthodontic and ortho- 
pedic treatment and general expertise for consultation with all of the other members of the cleft team. Different phases of active treatment will be necessary from birth to adulthoods. Orthodontist should consider many factors in determining when to initiate orthodontic treatment. These factors include the ability of the patient to cooperate, the severity of the malocclusion, the amount of jaw discrepancy, type of dental anomalies, existence of missing, dental shape anomalies, supernumerary teeth, and the need for future orthodontic treatment in the early mixed or permanent dentitions.

Orthodontic treatment plan can be divided into following stages based on the time of treatment:

(1) Presurgical orthopedics

(2) During primary dentition

(3) During mixed dentition

(4) During permanent dentition

\subsubsection{Presurgical orthopedics}

Presurgical infant orthopedics is sometimes used to relocate the segments of the cleft in maxilla prior to lip repair. A custom-fitted orthodontic appliance is applied to bring the parts of the lips, upper jaw, and nose closer together. This is called Nasoalveolar Molding (NAM). These appliances can make lip closure easier.

Advantages of presurgical orthopedics:

(a) To facilitate feeding

(b) To help establish normal tongue

(c) To provide psychological boost to the patients

(d) To assist surgeon in the initial repair

(e) To stimulate palatal growth and orofacial functional matrix

(f) To help decrease the number of ear infections

(g) Improve esthetics

(h) Repositioning of premaxilla

Although the evidence does not support the neonatal maxillary orthopedics as an essential or desirable routine procedure; Nonetheless, the molding of the segments achieved by these appliances does make definitive lip repair easier for the surgeon, especially for patients with a severely protruding premaxilla caused by a bilateral cleft lip [26].

\subsubsection{During primary dentition}

Midfacial deficiency is a common feature of cleft lip and palate patients due to scar tissue of the lip and palate closure [25]. During deciduous dentition, no orthodontic and orthopedic 
treatments are given because it has limited advantage. Orthodontic and orthopedic intervention starts in the mixed dentition.

\subsubsection{During mixed dentition}

Early orthopedic treatment in cleft palate children is essential because the maxillary bones and their component parts may be moved and altered in young children with relative ease and thereby creates a more functional dental arch. Orthodontic interventions in patients with cleft palate are focused at correction of Class III skeletal growth pattern, maxillary arch expansion, correction of upper incisor misalignments, gross rotations of incisors, and cross bite of buccal segments. Maxillary deficiency may be a reflection of the underlying skeletal abnormality for which growth modification and redirection may be indicated with a protraction headgear. Treatment approach to improve the midface deficiency was achieved by using the face mask $[2,27]$, tongue appliance [6], tongue plate [27], protraction head gear [3, 28], suborbital protraction appliances [4], ankylosed teeth [29], endosseous implants [30], and surgically assisted orthopedic protraction and distraction osteogenesis [8]. Jamilian et al. [6] evaluated the effectiveness of tongue appliance on deficient maxilla in growing cleft lip and palate patients. They showed that tongue appliance improved the deficient maxilla.

Tongue appliance has Adams clasp in first maxillary molars and C clasps in the upper incisors and deciduous teeth in order to increase the retention. A screw is mounted in midpalatal area to correct bilateral posterior crossbite. It was activated twice a week by the patient. Four separate tongue cribs were incorporated in the palatal area, behind the upper incisors. These cribs are long enough to cage the tongue and are adjusted in the clinic to avoid traumatizing the floor of the mouth. Tongue appliance is seen in Figure 1. Figure 2 shows the profile of the patient before application of the tongue appliance and Figure 3 shows the profile of the same patient after using the appliance. Extraoral appliances are not used suitably by the patients, whereas they prefer to apply small-sized and more convenient appliances. Besides to their large size of extraoral appliances, they need high compliance and many clinicians observe lack of cooperation by patients treated by big extraoral appliances. The philosophy of tongue appliance [31-33] is provided in the following two ways:

(1) The force of the tongue during each swallowing might be 5 pounds. Each patient might have 500-1200 times swallowing in 24 hours. The pressure from the tongue is transferred through the tongue appliance to the deficient maxilla.

(2) There is substantial continuous pressure of tongue. Because tongue is caged behind the cribs that is why this force is continuous in the rest position and centric occlusion. This force pushes the nasomaxillary complex into a forward position. In other words, functional activity and physiological position of tongue create these considerable forces that are conducted by tongue through the palatal cribs and finally transmitted to the deficient maxilla and nasomaxillary complex.

The more anterior the tongue, the greater pressure will be. The more posterior the crib, the greater pressure will be. Jamilian et al. [6] reported that the anterior part of maxillary plane moved superiorly (anteinclination) and posterior part of it relocated inferiorly. In other words, the maxillary posterior segment is extruded slightly and that is why the lower jaw 
rotated in a clockwise direction. These changes led to correction of the overjet and reducing of SNB and increasing of mandibular plane angle. Click wise rotation of the mandible is not favorable in long face patients.

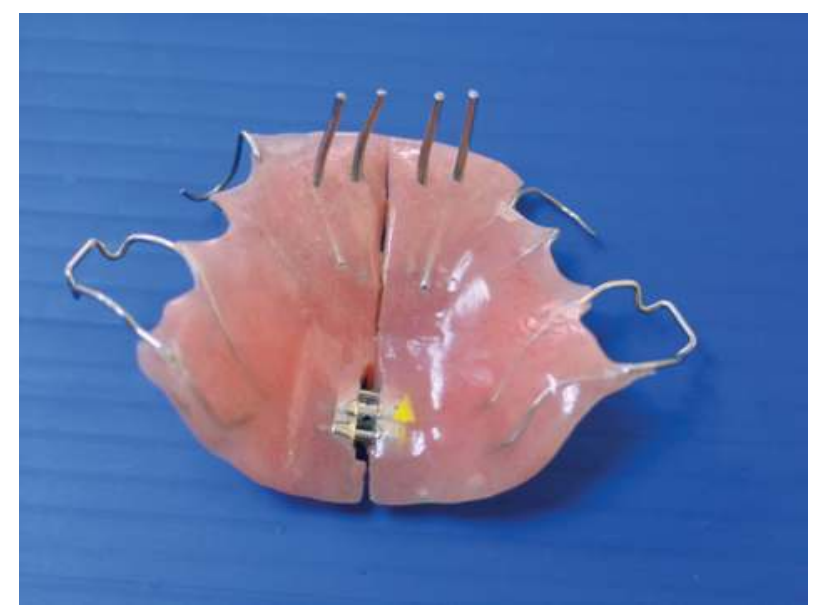

Figure 1. Tongue appliance with expansion screw.

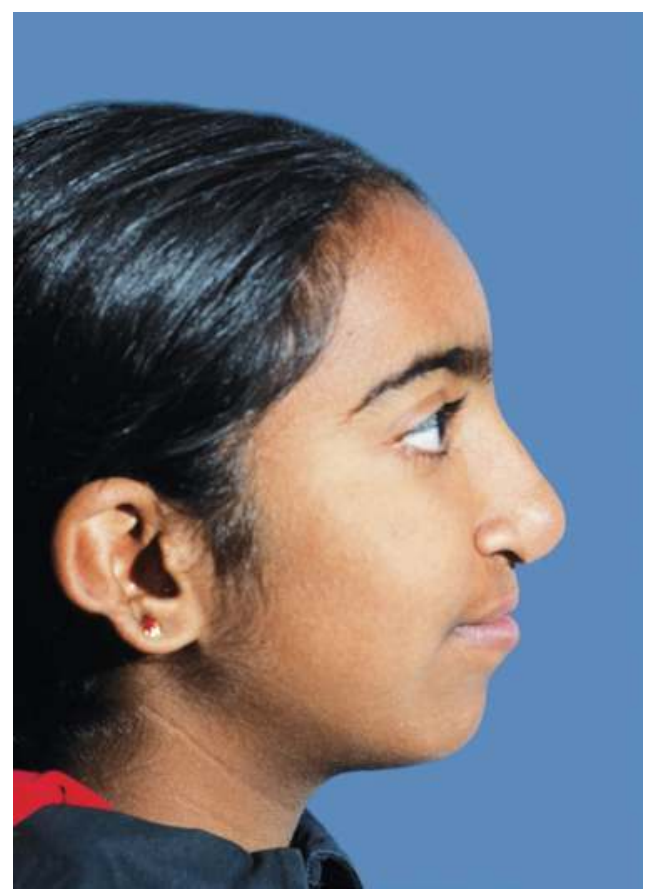

Figure 2. Profile view before treatment. 


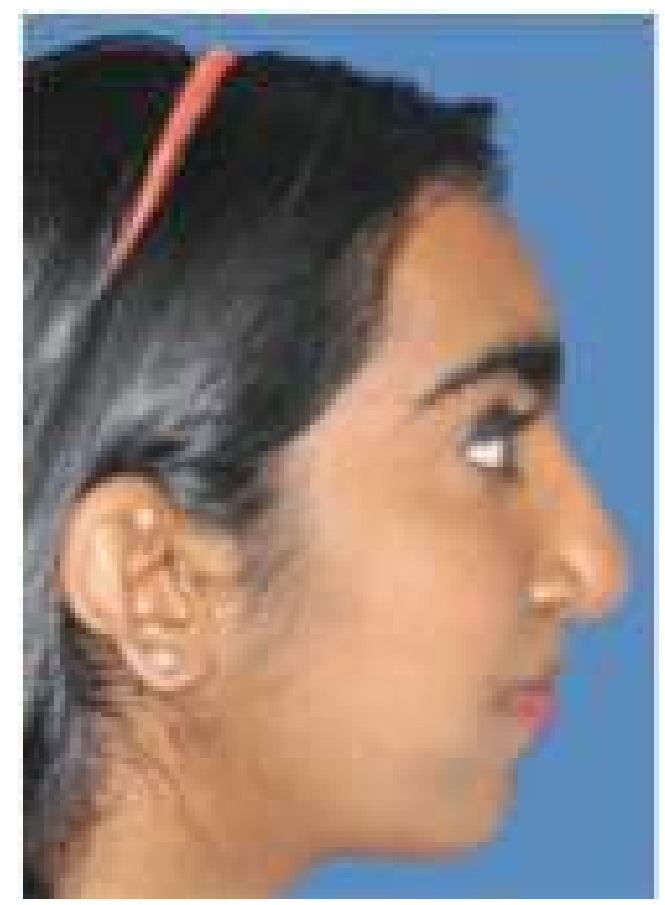

Figure 3. Profile view after treatment.

Tongue plate has been used for correction of maxillary deficiency [27]. Mechanism of tongue plate [7] is very similar to the tongue appliance. The pressure of tongue during rest position and swallowing is transmitted through the tongue plate to the deficient maxilla. The significant pressure of the tongue that is caged behind the acrylic plate transfers the nasomaxillary complex in a forward position. Some patients had some extent of irritation on the tongue from tongue appliance that is why tongue appliance was replaced by tongue plate. This has a smoothed area and these softened edges create it undamaging for the patient. Moreover, it is adjusted in a way to avoid traumatizing the floor of the mouth. Elimination of the cribs in tongue plate might offer a better psychological sense to the patients. Extra oral appliances such as facemask and reverse chin cup $[5,34]$ improve the deficient nasomaxillary complex but they have an unfavorable effect on the normal mandible. Extra oral appliances rotate the mandible in clockwise rotation and it is unfavorable in normal growth pattern and long face patients. Besides, the cup of facemask can create abrasion particularly in warm climates. Tongue appliance is an intraoral appliance and it is very comfortable and simple. It is constructed easily and it is not expensive. Tongue appliance and tongue plate will be accepted better than extraoral appliances due to less conspicuous of them. Children with cleft lip and palate have suffered from birth; tongue plate and tongue appliance are more comfortable as they create the least stress to patients in comparison with other big extraoral appliances. In spite of many advantages of them, tongue appliance and tongue plate have one disadvantage. 
Incisor mandibular angle is reduced due to removal of the pressure of tongue to the lower incisors and acting force of orbicularis oris to them. Tongue appliance and tongue plate have a screw to correct bilateral posterior crossbite. Expansion will lose all maxillary sutures like zygomaxillary, pterygomaxillary, thus, maxilla will transfer more easily in forward direction $[35,36]$. On the other hand, upper jaw may be expanded in order to improve dental function, reliving crowding, align upper incisors, eliminate functional shifts, provide access for impacted teeth in the cleft site, provide restorative treatment to carious teeth, improve maxillary deficiency, and nasal airway in mixed dentition [6,31]. Maxillary skeletal asymmetry in unilateral lip and palate patients may be reflected in a unilateral posterior cross bite, which may be corrected with a removable appliance with a screw or a quad helix type of appliance in the mixed dentition. A bonded or non-bonded hyrax expander are recommended along with extra oral traction, such as facial mask to stimulate maxillary protraction or chin cup to control mandibular growth (Figures 4 and 5). Patients with maxillary cleft do not have a midpalatal suture, which requires orthopedic forces to open. Instead there is a midpalatal cleft covered with scarred and repaired palatal tissues limiting the rate and amount of expansion. The slower rate of expansion and the lower force magnitude provided by a quad helix appliance allows the soft tissue of the palate to the increasing maxillary width, avoiding a breakdown of the scar tissue that can result in an oronasal fistula. As the permanent incisors erupt adjacent to the cleft site, maxillary incisors typically are rotated, misplaced, malformed, or hypo plastic. Removable appliance is used to correct upper incisor misalignment. Moreover, incisors may be absent or peg shaped and there may be one or some supernumerary teeth. Maxillary expansion appliances can be anchored on the permanent first molars and extended anteriorly to improve maxillary arch while correcting the cross bite. Usually expansion is followed by maxillary protraction using a traditional Delaire or other protraction appliances. After the expansion and protraction phase, full fixed appliances along with Class III elastics and sometimes extractions, if necessary, are usually needed to correct the malocclusion [33]. Figures 6 and 7 show the intraoral photograph of a patient before and after orthodontic treatment, respectively. Figures 8 and $\mathbf{9}$ show the frontal view of the same patient in pre- and postorthodontic treatment, respectively.

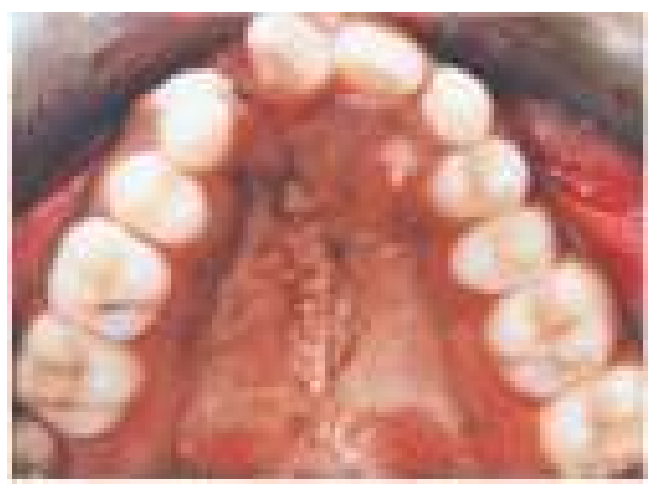

Figure 4. Upper arch before expansion. 


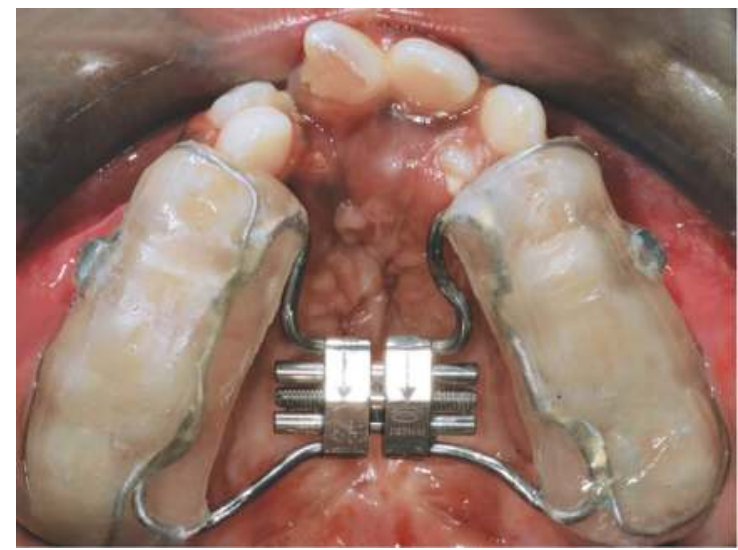

Figure 5. Upper arch during expansion with bonded hyrax.

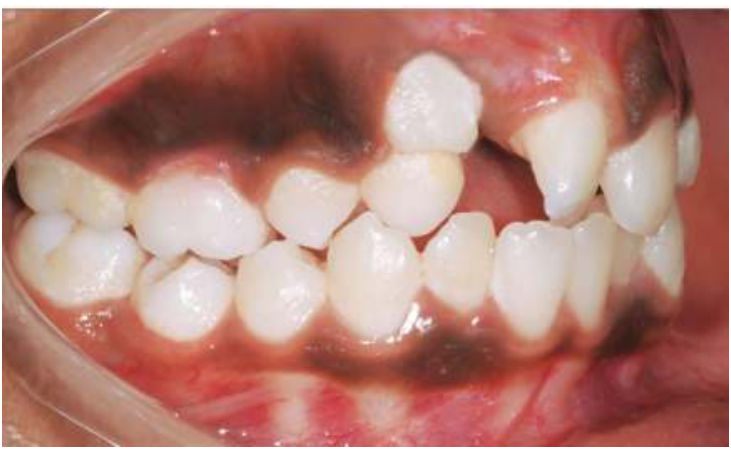

Figure 6. Intraoral view before orthodontic treatment.

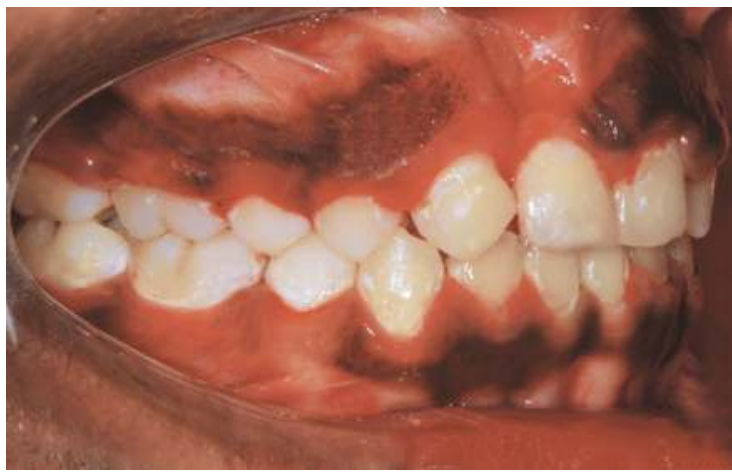

Figure 7. Intraoral view after orthodontic treatment. 


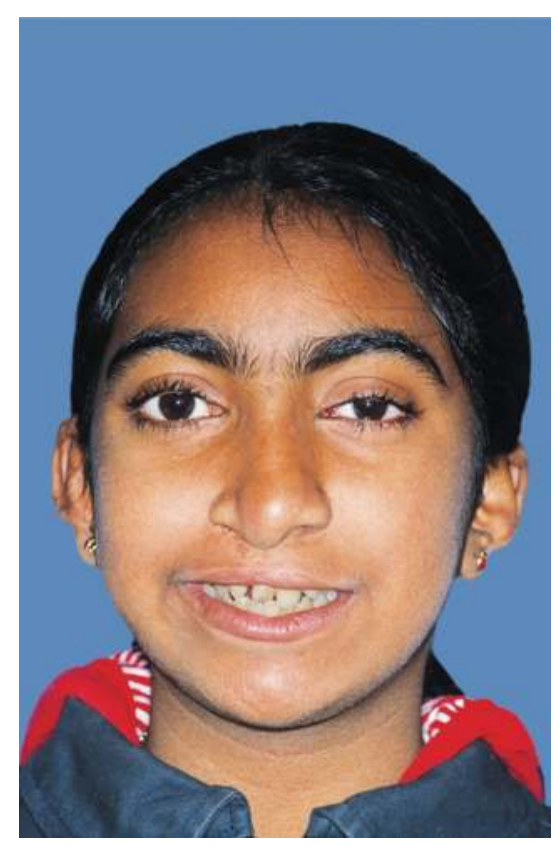

Figure 8. Frontal view before treatment.

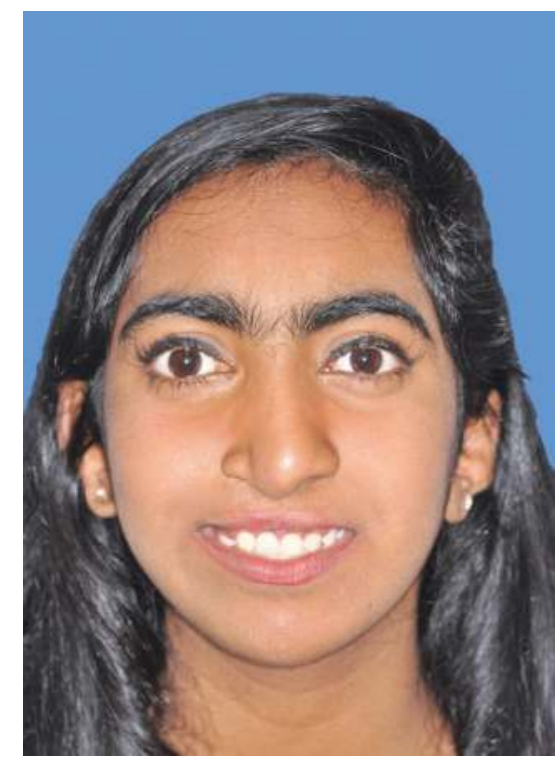

Figure 9. Frontal view after treatment. 


\subsubsection{Permanent dentition}

Comprehensive fixed appliance therapy usually occurs in the permanent dentition with the aim of preparing for alveolar bone graft that can be done by an oral surgeon. This phase usually involves aligning of malposed maxillary incisors. Reverse pull headgear or face mask therapy, expansion of maxillary arch may be continued during this time period. Final alignment of teeth is carried over with or without extraction. Orthodontic management is limited after eruption of permanent dentition. The established malocclusion and discrepancy between the upper and lower arch often require orthognathic surgery.

\subsection{Role of oromaxillofacial surgeon in cleft lip and palate patients}

Orthognathic procedure may be designated if a malocclusion develops due to abnormal growth of the maxilla. Treatment for adults may involve surgical in severe or camouflage in mild to moderate patients. Orthognathic surgery is performed to patients with deformities of the jaws to improve facial esthetics as well as to correct dental occlusion. Presurgical orthodontics are usually necessary to align the teeth, correct any compensations, decrowding, eliminate any dental midline discrepancy, coordinate arches, and localize space for prosthetic or implant replacement of the teeth. Ideally, the patient is referred to the surgeon after presurgical orthodontics. The postsurgical phase of orthodontics is required for creating perfect occlusion and better interdigitation after surgery.

\subsubsection{Pharyngeal flap}

Failure to achieve a seal between the posterior pharyngeal wall and soft palate lets leakage of air through the nose and causes cleft palate speech. This has proved not to be a problem in patients with a normal velopharyngeal mechanism. If a patient has speech problems a surgical procedure to create a pharyngeal flap probably will be required. To correct hyper nasality, this procedure contains raising a flap of tissue from the posterior pharyngeal wall and incorporating it into the soft palate. Pharyngeal flap is used when the muscles do not function appropriately or the repaired palate is too short. For adults with speech problems, pharyngeal flap, combined with an intensive schedule of speech therapy, can produce noticeable improvements.

\subsubsection{Distraction osteogenesis}

Distraction osteogenesis has become a new method for correction of maxillary deficiency [8, 37]. Distraction osteogenesis for maxillary advancement started in 1993 and is now broadly used in cases with skeletal Class III deformity due to maxillary deficiency [38]. Figueroa and Polley [39] reported that distraction osteogenesis was successfully used to advance the maxilla in children with cleft lip and palate. According to them the main advantages of distraction osteogenesis compared with conventional methods of craniofacial reconstruction were reduced time of surgery and cost and the ability to generate new bone. Many advantages and disadvantages of distraction devices have been detailed. For a large advancement in a patient with a cleft lip or palate, distraction osteogenesis may be advantageous. Figueroa and Polley [39] assessed the cephalometric landmarks on 14 patients with cleft palate who 
were treated with a rigid external distraction technique. Distraction osteogenesis provides less physical and psychological invasion in comparison to conventional LeFort osteotomy: that is, reduced operating time, less blood loss, less postoperative pain, and shorter hospitalization. Also, when intra-arch distraction is applied to lengthen the mandible or maxilla, orthodontic alignment of upper and lower arches are not necessary before the procedure [40]. Intraoral distraction osteogenesis devices are divided into two types namely bone borne or tooth borne. Advantages of these are their smaller size and better patient acceptance; however, the bone attached one has the disadvantage of the need for second intervention to remove the device [41].

Hyrax screw incorporated in an acrylic plate has been applied for treatment of maxillary deficient in cleft lip and palate patients. After creating horizontal cuts similar to LeFort 1 and vertical cuts between the premolars on both sides, a bonded hyrax screw was mounted on an acrylic plate for the slow sagittal expansion of upper arch. The distraction procedure can be initiated after 5 days of latency period.

The expansion is performed by activating the hyrax screw $0.8 \mathrm{~mm}$ per day after the latency period (Figure 10). Expansion was discontinued after achieving satisfactory overjet and occlusion (Figure 11). If open bite occurs during expansion period vertical elastics will be used to correct open bite. Consolidation period lasts 8 weeks and after this period hyrax screw is removed. One of the advantages of anterior maxillary distraction is that velopharyngeal area will be intact. Anterior distraction is used for skeletal deformity and fixed appliance is applied for correction of dental problems [8].

Some researchers suggested that distraction orthogenesis does not seem to have any advantages over surgery. Additionally, the occlusion at the end of distraction is much less defined than what is seen with conventional orthognathic surgery [42].

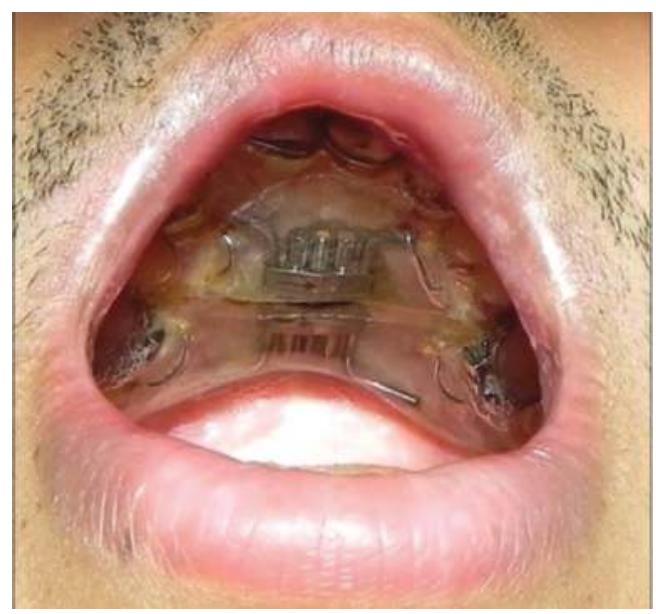

Figure 10. Hyrax screw incorporated in an acrylic plate. 


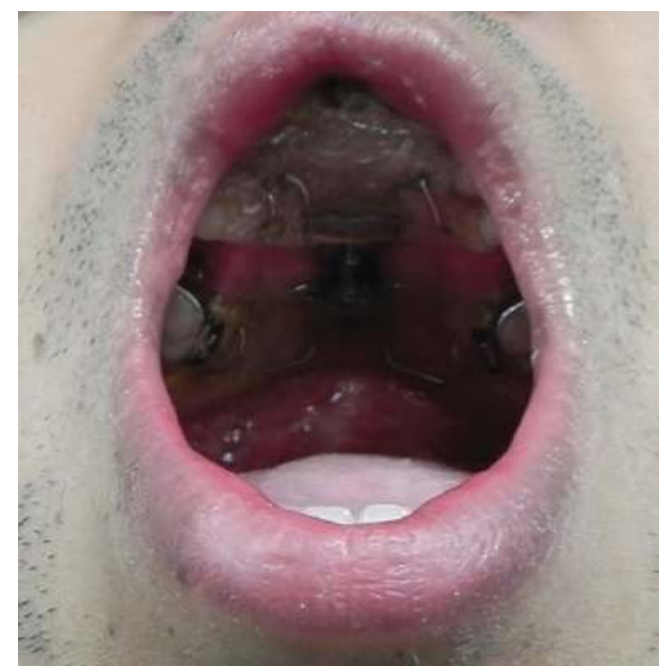

Figure 11. Hyrax screw after activation.

\section{Retention}

The retention protocol for clefts follows the same principles of orthodontic treatment in Class III malocclusion without clefts. The upper and lower arches should have been coordinated throughout orthodontic and orthopedic intervention. Positive overjet and overbite with adequate intercuspation are necessary for retention. Hawley retainer in the maxillary and mandibular arch is used for retention. Hawley appliance often includes prosthetic teeth that will be displaced later with dental implants or prostheses. Patients are instructed to wear the Hawley appliance for 12 months continuously. After that, if the occlusion is stable, the Hawley retainer is used at night-time for additional six months.

Retention in cleft palate cases is longer than for noncleft patients. The reasons are due to:

(a) lack of bony stability,

(b) contracture of stretched or scar tissues, and

(c) missing teeth.

\section{Recommendation}

Treatment approach depends on the age and severity of the patients. Growth modification is the best treatment plan when the patient is still growing. We can take advantages of a child's growing years by guiding proper jaw bone formation with small intraoral appliances such as tongue plate and tongue appliance $[6,27]$. These small intraoral appliances are recommended 
in cleft lip and palate patients who suffering from psychological and social problems associated with their malocclusion in early mixed children. Once the patient is an adult, camouflage may be an option for correcting mild deformities and surgery would be proper treatment plan for severe cases.

\section{Conclusion}

Cleft is the most common craniofacial malformation that an orthodontist may encounter. The orthodontist's role in the cleft lip and palate team requires close relationship with the other team members. Cleft lip and palate patients become more maxillary deficient and mandibular prognathic in their appearance. The most common specialties involved in the care of a child with a cleft are: oromaxillofacial surgeon, plastic surgeon, psychologist, orthodontist, general dentist, otolaryngologist, speech therapist, pediatrician, and prosthodontist.

Treatment plan from orthodontic perspective can be divided into the following stages based on the dentition stages: (1) presurgical orthopedics, (2) primary dentition, (3) mixed dentition, and (4) permanent dentition.

\section{Author details}

Letizia Perillo ${ }^{1}$, Fabrizia d'Apuzzo ${ }^{1}$, Sara Eslami² and Abdolreza Jamilian²*

*Address all correspondence to: info@jamilian.net

1 Multidisciplinary Department of Medical-Surgical and Dental Specialties, Second University of Naples, Naples, Italy

2 Department of Orthodontics, Cranio Maxillofacial Research Center, Tehran Dental Branch, Islamic Azad University, Tehran, Iran

\section{References}

[1] Jamilian, A., F. Nayeri, and A. Babayan, Incidence of cleft lip and palate in Tehran. J Indian Soc Pedod Prev Dent 2007;25:174-6.

[2] Buschang, P.H., et al., Face mask therapy of preadolescents with unilateral cleft lip and palate. Angle Orthod 1994;64:145-50.

[3] Chen, K.F. and L.L. So, Sagittal skeletal and dental changes of reverse headgear treatment in Chinese boys with complete unilateral cleft lip and palate. Angle Orthod 1996;66:363-72. 
[4] Delaire, J. and P. Verdon, The use of heavy postero-anterior extraoral forces by an orthopedic mask in the treatment of dentomaxillary sequellae of labiomaxillopalatal clefts. Chir Pediatr 1983;24:315-22.

[5] Showkatbakhsh, R. and A. Jamilian, A novel approach in treatment of maxillary deficiency by reverse chin cup. Int J Orthod Milwaukee 2010;21:27-31.

[6] Jamilian, A., R. Showkatbakhsh, and M.B. Boushehry, The effect of tongue appliance on the nasomaxillary complex in growing cleft lip and palate patients. J Indian Soc Pedod Prev Dent 2006;24:136-9.

[7] Showkatbakhsh, R. and A. Jamilian, A novel method of maxillary deficiency treatment by tongue plate-a case report. Int J Orthod Milwaukee 2011;22:31-4.

[8] Srivastava, D., et al., Use of anterior maxillary distraction osteogenesis in two cleft lip and palate patients. Natl J Maxillofac Surg 2015;6:80-3.

[9] Rullo, R., et al., Speech outcome in unilateral complete cleft lip and palate patients: a descriptive study. Eur J Paediatr Dent 2014;15:293-6.

[10] Grandjean, H., D. Larroque, and S. Levi, The performance of routine ultrasonographic screening of pregnancies in the Eurofetus Study. Am J Obstet Gynecol 1999;181:446-54.

[11] Lourenco Ribeiro, L., et al., Dental anomalies of the permanent lateral incisors and prevalence of hypodontia outside the cleft area in complete unilateral cleft lip and palate. Cleft Palate Craniofac J 2003;40:172-5.

[12] Akcam, M.O., et al., Dental anomalies in individuals with cleft lip and/or palate. Eur J Orthod 2010;32:207-13.

[13] Shapira, Y., E. Lubit, and M.M. Kuftinec, Hypodontia in children with various types of clefts. Angle Orthod 2000;70:16-21.

[14] Vichi, M. and L. Franchi, Abnormalities of the maxillary incisors in children with cleft lip and palate. ASDC J Dent Child 1995;62:412-7.

[15] Larson, M., R. Hellquist, and O.P. Jakobsson, Dental abnormalities and ectopic eruption in patients with isolated cleft palate. Scand J Plast Reconstr Surg Hand Surg 1998;32:203-12.

[16] Jiroutova, O. and Z. Mullerova, The occurrence of hypodontia in patients with cleft lip and/or palate. Acta Chir Plast 1994;36:53-6.

[17] Paranaiba, L.M., et al., Prevalence of dental anomalies in patients with nonsyndromic cleft lip and/or palate in a Brazilian population. Cleft Palate Craniofac J 2013;50:400-5.

[18] Tortora, C., et al., Prevalence of abnormalities in dental structure, position, and eruption pattern in a population of unilateral and bilateral cleft lip and palate patients. Cleft Palate Craniofac J 2008;45:154-62.

[19] Menezes, R. and A.R. Vieira, Dental anomalies as part of the cleft spectrum. Cleft Palate Craniofac J 2008;45:414-9. 
[20] Laatikainen, T. and R. Ranta, Hypodontia in twins discordant or concordant for cleft lip and/or palate. Scand J Dent Res 1994;102:88-91.

[21] Polder, B.J., et al., A meta-analysis of the prevalence of dental agenesis of permanent teeth. Community Dent Oral Epidemiol 2004;32:217-26.

[22] Shapira, Y., E. Lubit, and M.M. Kuftinec, Congenitally missing second premolars in cleft lip and cleft palate children. Am J Orthod Dentofacial Orthop 1999;115:396-400.

[23] Ranta, R., A review of tooth formation in children with cleft lip/palate. Am J Orthod Dentofacial Orthop 1986;90:11-8.

[24] Pope, A.W., Points of risk and opportunity for parents of children with craniofacial conditions. Cleft Palate Craniofac J 1999;36:36-9.

[25] Hunt, O., et al., The psychosocial effects of cleft lip and palate: a systematic review. Eur J Orthod 2005;27:274-85.

[26] Vig, K.W. and T.A. Turvey, Orthodontic-surgical interaction in the management of cleft lip and palate. Clin Plast Surg 1985;12:735-48.

[27] Showkatbakhsh, R., et al., The effects of face mask and tongue plate on maxillary deficiency in growing patients: a randomized clinical trial. J Orthod 2013;40:130-6.

[28] Showkatbakhsh, R., et al., The effects of face mask and tongue appliance on maxillary deficiency in growing patients: a randomized clinical trial. Prog Orthod 2012;13:266-72.

[29] Omnell, M.L. and B. Sheller, Maxillary protraction to intentionally ankylosed deciduous canines in a patient with cleft palate. Am J Orthod Dentofacial Orthop 1994;106:201-5.

[30] Smalley, W.M., et al., Osseointegrated titanium implants for maxillofacial protraction in monkeys. Am J Orthod Dentofacial Orthop 1988;94:285-95.

[31] Jamilian, A. and R. Showkatbakhsh, The effect of tongue appliance on the maxilla in Class III malocclusion due to maxillary deficiency. Int J Orthod Milwaukee 2009;20:11-4.

[32] Showkatbakhsh, R., et al., Maxillary deficiency treatment by fixed tongue appliance-a case report. Int J Orthod Milwaukee 2013;24:31-4.

[33] Showkatbakhsh, R., et al., The short-term effects of face mask and fixed tongue appliance on maxillary deficiency in growing patients - a randomized clinical trial. Int J Orthod Milwaukee 2015;26:33-8.

[34] Showkatbakhsh, R., et al., The effects of facemask and reverse chin cup on maxillary deficient patients. J Orthod 2012;39:95-101.

[35] Liou, E.J. and W.C. Tsai, A new protocol for maxillary protraction in cleft patients: repetitive weekly protocol of alternate rapid maxillary expansions and constrictions. Cleft Palate Craniofac J 2005;42:121-7.

[36] Showkatbakhsh, R., et al., Bone remodeling to correct maxillary deficiency after growth cessation. Natl J Maxillofac Surg 2012;3:202-6. 
[37] Polley, J.W. and A.A. Figueroa, Rigid external distraction: its application in cleft maxillary deformities. Plast Reconstr Surg 1998;102:1360-72; discussion 1373-4.

[38] Kuroda, S., et al., Maxillary distraction osteogenesis to treat maxillary hypoplasia: comparison of an internal and an external system. Am J Orthod Dentofacial Orthop 2005; 127:493-8.

[39] Figueroa, A.A. and J.W. Polley, Management of severe cleft maxillary deficiency with distraction osteogenesis: procedure and results. Am J Orthod Dentofacial Orthop 1999; 115:1-12.

[40] Dessner, S., et al., Mandibular lengthening using preprogrammed intraoral tooth-borne distraction devices. J Oral Maxillofac Surg 1999;57:1318-22; discussion 1322-3.

[41] Iannetti, G., et al., LeFort III advancement with and without osteogenesis distraction. J Craniofac Surg 2006;17:536-43.

[42] Van Sickels, J.E., Distraction osteogenesis versus orthognathic surgery. Am J Orthod Dentofacial Orthop 2000;118:482-4. 